\title{
Periinsular anterior quadrantotomy: technical note
}

\author{
Giulia Cossu, MD, ${ }^{1}$ Sebastien Lebon, MD, ${ }^{2}$ Margitta Seeck, MD, ${ }^{3}$ Etienne Pralong, MD, ${ }^{1}$ \\ Mahmoud Messerer, MD, MSc, ${ }^{1}$ Eliane Roulet-Perez, MD, ${ }^{2}$ and Roy Thomas Daniel, MBBS, MCh$^{1}$
}

\begin{abstract}
${ }^{1}$ Department of Neurosurgery and 2 Unit of Pediatric Neurology and Neurorehabilitation, Department of Pediatrics, University Hospital of Lausanne; and ${ }^{3}$ Epilepsy Unit, Department of Neurology, University Hospital of Geneva, Switzerland
\end{abstract}

\begin{abstract}
Refractory frontal lobe epilepsy has been traditionally treated through a frontal lobectomy. A disconnective technique may allow similar seizure outcomes while avoiding the complications associated with large brain resections. The aim of this study was to describe a new technique of selective disconnection of the frontal lobe that can be performed in cases of refractory epilepsy due to epileptogenic foci involving 1 frontal lobe (anterior to the motor cortex), with preservation of motor function. In addition to the description of the technique, an illustrative case is also presented.

This disconnective procedure is divided into 4 steps: the suprainsular window, the anterior callosotomy, the intrafrontal disconnection, and the frontobasal disconnection. The functional neuroanatomy is analyzed in detail for each step of the surgery. It is important to perform cortical and subcortical electrophysiological mapping to guide this disconnective procedure and identify eloquent cortices and intact neural pathways.

The authors describe the case of a 9-year-old boy who presented with refractory epilepsy due to epileptogenic foci localized to the right frontal lobe. MRI confirmed the presence of a focal cortical dysplasia of the right frontal lobe. A periinsular anterior quadrant disconnection (quadrantotomy) was performed. The postoperative period was uneventful, and the patient was in Engel seizure outcome Class I at the 3-year follow-up. A significant cognitive gain was observed during follow-up.

Periinsular anterior quadrantotomy may thus represent a safe technique to efficiently treat refractory epilepsy when epileptogenic foci are localized to 1 frontal lobe while preserving residual motor functions.

https://thejns.org/doi/abs/10.3171/2017.8.PEDS17339
\end{abstract}

KEY WORDS anterior hemi-hemispherotomy; anterior quadrantotomy; disconnection technique; epilepsy surgery; hemimegalencephaly; refractory epilepsy; surgical technique

$\mathrm{H}$ EMISPHEROTOMY is an excellent surgical option in cases of multifocal epilepsy restricted to 1 hemisphere associated with a complete or near-complete loss of function in this hemisphere. ${ }^{25}$ When the epilepsy is restricted to one-half of the hemisphere (anterior or posterior quadrant), disconnective surgery of the involved quadrant becomes the surgical objective. We previously reported the technique of posterior quadrantotomy for the disconnection of the temporal, occipital, and parietal lobes in 1 hemisphere. ${ }^{7}$ Here, to our knowledge, we present the first report of the surgical technique of performing an anterior quadrant disconnection (quadrantotomy), namely the disconnection of the frontal lobe anterior to the primary motor cortex, when the epileptogenic foci are restricted to one frontal lobe and residual motor functions persist.

\section{Operative Technique}

After induction of general anesthesia, the patient is placed in the dorsal decubitus position, with the head fixed in a 3-pin Mayfield head holder and turned to the opposite side. The ipsilateral shoulder is elevated with a cushion. A frontotemporal "barn door" incision is made, extending to about $1.5 \mathrm{~cm}$ from the midline. The cutaneous flap and

ABBREVIATIONS EEG = electroencephalography; IFG = inferior frontal gyrus; IFOF = inferior frontoocipital fasciculus; MFG = middle frontal gyrus; $S F G=$ superior frontal gyrus.

SUBMITTED June 19, 2017. ACCEPTED August 24, 2017.

INCLUDE WHEN CITING Published online December 8, 2017; DOI: 10.3171/2017.8.PEDS17339. 


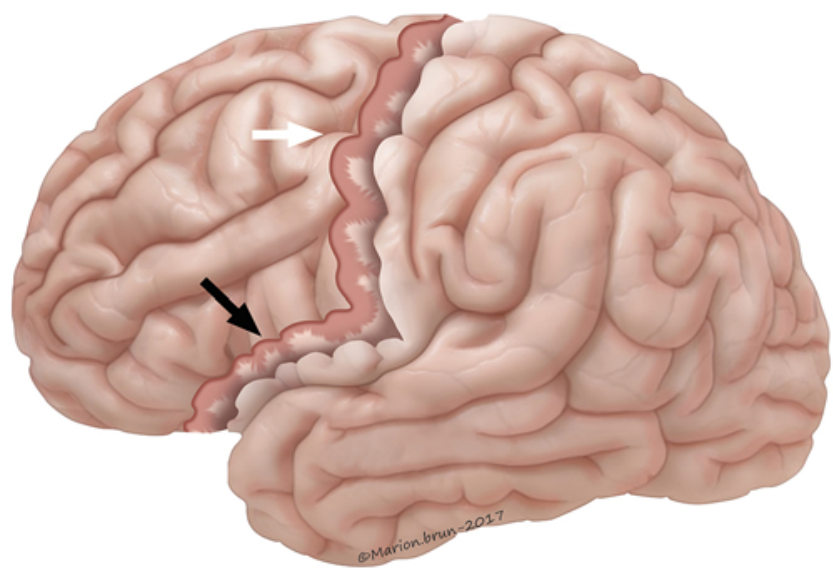

FIG. 1. Schematic representation of the anterior quadrantotomy, showing the disconnection at the level of the IFG through the pars orbitalis, pars triangularis, and pars opercularis (first portion of the suprainsular window, black arrow) and the intrafrontal disconnection (just anterior to the motor cortex), through the posterior parts of the IFG, MFG, and SFG (white arrow). Copyright Marion Brun-Baud. Published with permission. Figure is available in color online only.

superior portion of the temporal muscle are elevated and retracted. A large fronto-temporo-parietal craniotomy is performed, and, after elevation of the bone flap, the dura is suspended at the periphery of the craniotomy. The dura is opened in a rectilinear fashion, thus allowing a wide exposition of the sylvian fissure, periinsular and perirolandic cortices.

\section{Mapping of the Perirolandic Cortices}

The primary motor and sensory cortices are localized initially through visual inspection by examining the sulcogyral anatomy of the exposed cortices together with the preoperative cerebral MR image. At the cortical level, the following structures should be identified: the superior frontal sulcus, which separates the superior frontal gyrus (SFG) and the middle frontal gyrus (MFG), and joins the precentral sulcus at its posterior edge in a perpendicular fashion. The precentral gyrus is thus identified posterior to the precentral sulcus. Then, the inferior frontal sulcus separates the MFG from the inferior frontal gyrus (IFG). This last gyrus is divided into orbital, triangular, and opercular portions by the horizontal and ascending anterior sulcus of the sylvian fissure.

The central sulcus is confirmed through electrophysiological means. A quadripolar subdural platinum electrode (AD-TECH) is positioned over the cerebral cortex. The phase inversion between the frontal (P22) and the parietal (N20) response to the contralateral median nerve $(3.7 \mathrm{~Hz}$, $10 \mathrm{~mA}, 200 \mu \mathrm{sec}$ ) stimulation indicates that the electrodes are on either side of the central sulcus. Contralateral motor response to cortical anodic stimulation (train-of-five, 500 $\mathrm{Hz}, 200 \mu \mathrm{sec}$, monopolar, $5 \mathrm{~mA}$ ) confirms location of the motor cortex.

The same protocol is used for white matter stimulation, with the stimulation switched from anodic to cathodic to detect and prevent damage to motor corona radiata fibers. In addition, continuous somatosensory evoked potential

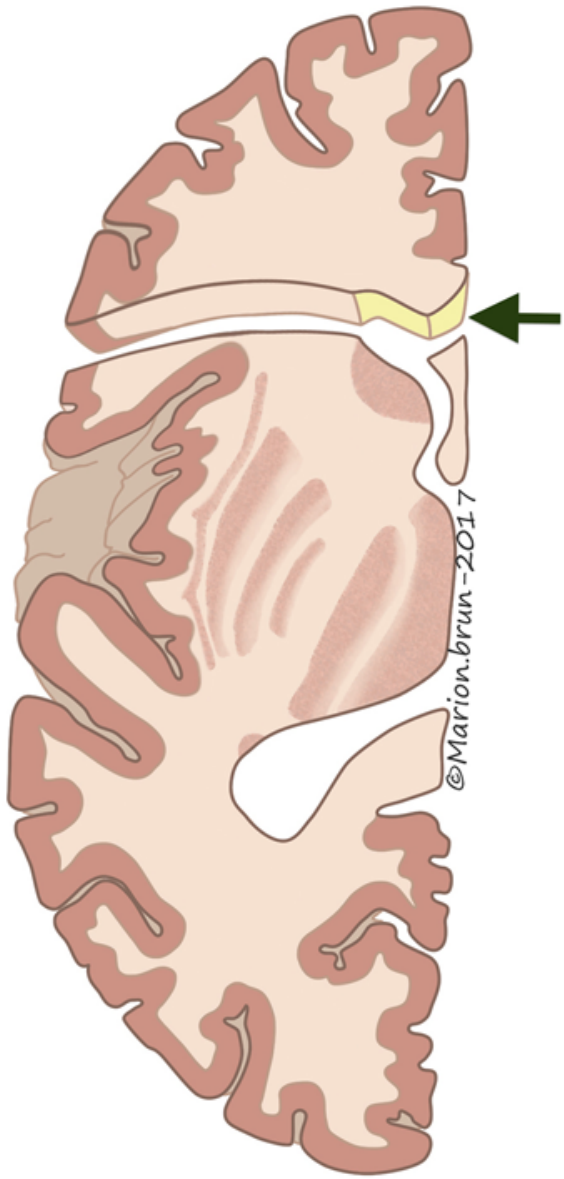

FIG. 2. Cross-sectional illustration of the procedure showing the section of the anterior portion of the internal capsule (anterior isthmus) that terminates in the frontal horn anterior to the head of the caudate nucleus. The diagram also shows the position of the anterior callostomy in the parasagittal plane (arrow). Copyright Marion Brun-Baud. Published with permission. Figure is available in color online only.

monitoring is performed $;^{1}$ these potentials should remain stable until the end of the disconnection.

\section{Surgical Steps}

The disconnection procedure is divided into the following steps.

\section{Anterior Suprainsular Window}

The pia mater over the IFG is coagulated about $5 \mathrm{~mm}$ away from the sylvian fissure. This procedure is performed in an anteroposterior direction through the pars orbitalis, pars triangularis, and pars opercularis (Fig. 1). The frontal operculum is removed subpially to identify the superior half of the circular sulcus and insula. At the limit of the circular sulcus, an incision is made into the white matter in the direction of the frontal horn of the ipsilateral ventricle. The frontal horn is entered anterior to the head of the caudate nucleus (Fig. 2).

\section{Anterior Callosotomy}

An incision is placed within the ventricle at the junc- 


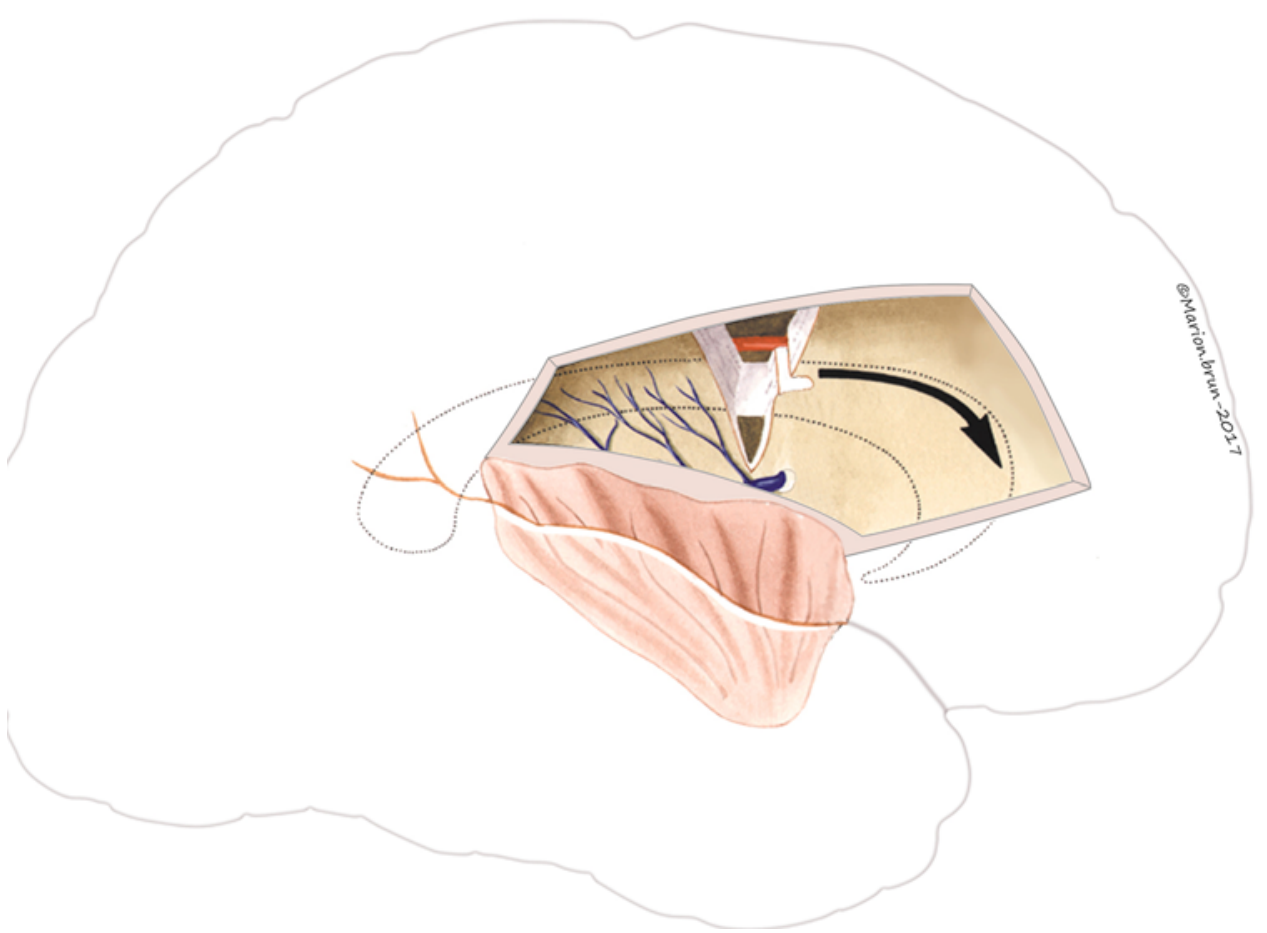

FIG. 3. Illustration of the anterior corpus callosotomy through the window into the anterior part of the lateral ventricle. The initial incision is made in a superoinferior direction at the junction of the septum pellucidum and roof of the ventricle. The pericallosal cistern and anterior cerebral arteries are identified, which are then followed in a posteroanterior direction to complete the anterior callosotomy (arrow). Copyright Marion Brun-Baud. Published with permission. Figure is available in color online only.

tion between the roof of the lateral ventricle and the septum pellucidum in a parasagittal direction (Fig. 2). The callosal fibers are sectioned so as to reach the pericallosal cistern. The pericallosal arteries are identified, and their exposure ensures the section of the corpus callosum. Anteriorly, the artery is followed until the $\mathrm{A}_{2}-\mathrm{A}_{3}$ junction to complete the disconnection at the genu and rostrum of the corpus callosum (Fig. 3). When cutting near the interventricular foramen, careful dissection must be performed to avoid inadvertent injury to the columns of the fornix.

The disconnection is stopped posteriorly when reaching the junction of the middle and last thirds of the body of the corpus callosum, thereby keeping intact the callosal fibers originating from the primary motor cortex and the parietal lobe.

\section{Intrafrontal Disconnection}

This disconnection is performed just anterior to the motor cortex. The incision starts at the operculum, just anterior to the precentral gyrus, and continues parallel to this gyrus, traversing the IFG, MFG, and SFG at their posterior portion (Fig. 1). Then, dissection of the white matter is continued until the ventricle is completely opened parallel to the cortical incision. Subsequently, the medial ventricular wall is incised to reach the interhemispheric fissure along its entire vertical dimension. This will section the cingulate gyrus and the mesial part of the SFG.

\section{Frontobasal Disconnection}

This part of the surgery disconnects the basal and inferomesial portions of the frontal lobe. The pial incision begins at the convexity of the frontal lobe, anterior to the opercular incision and is carried out along the direction of the sphenoid ridge. The white matter deep to this is disconnected. The white matter disconnection is continued onto the basal portion of the frontal lobe, keeping the basal pia intact in a subpial manner and thus crossing the orbital surface of the frontal lobe. At its posterior limit, the white matter incision is turned medially through the gyrus rectus in a subpial manner to visualize the mesial pia just anterior to the bifurcation of the internal carotid artery. The white matter incisions should reach the ventricle, where the posterior portion of the frontobasal disconnection reaches the level of the callosal disconnection at the rostrum (Fig. 4).

\section{Functional Neuroanatomy of the Periinsular Anterior Quadrantotomy Suprainsular Window}

The creation of the suprainsular window disconnects the following structures. 1) The anterior portion of the internal capsule (or anterior isthmus, between the anterior limiting sulcus and the lateral edge of the frontal horn) is disrupted, thus disconnecting the projection fibers represented by the anterior thalamic radiation and the frontopontine tract. 2) The superficial portion of the inferior frontoocipital fasciculus (IFOF), which connects the orbital and triangular partes of the IFG and occipital cortex, is disrupted. 3) The arcuate fasciculus, which encircles the insula connecting the superior temporal gyrus and the IFG and partially overlap with the IFOF fibers is also dis- 


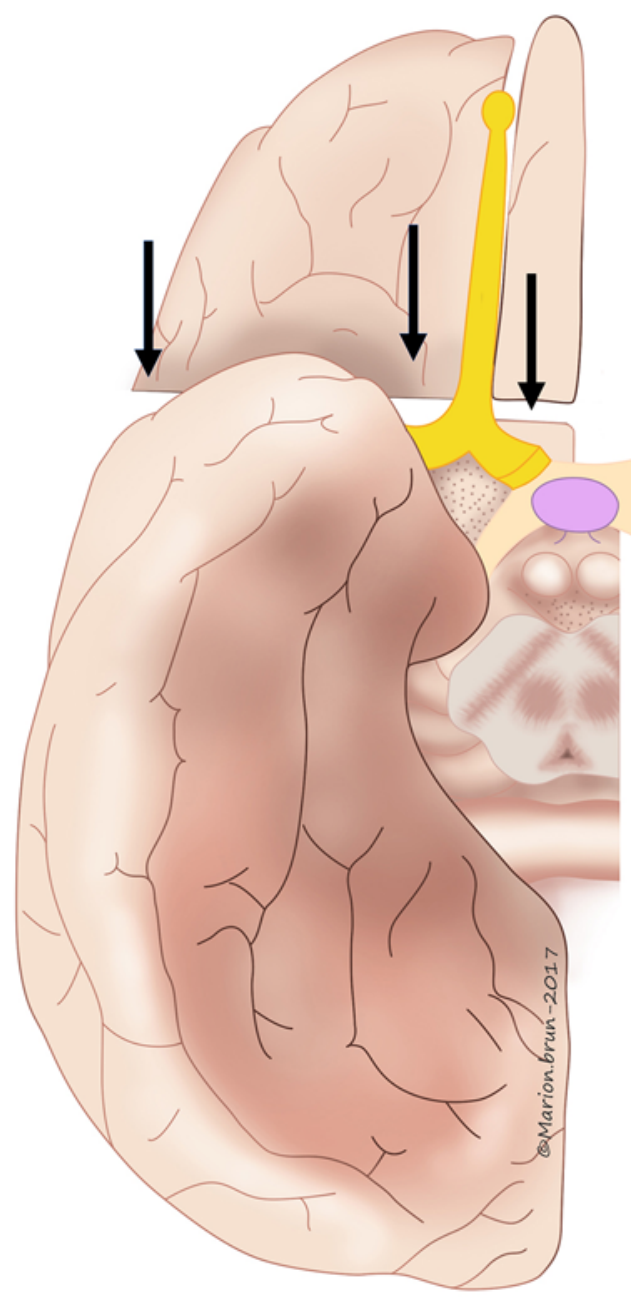

FIG. 4. Illustration of the frontobasal disconnection. The pial incision starts at frontal convexity brain (convexity pia) and proceeds parallel to the sphenoid ridge (left arrow). The white matter is interrupted deep to this incision. This disconnection proceeds onto the orbital surface of the frontal lobe in a subpial plane (basal pia) (center arrow). Subsequently, the white matter disconnection turns onto the mesial part of the frontal lobe in a subpial plane (mesial pia) (right arrow). The disconnection terminates at the level of the callosal disconnection of the rostrum. Copyright Marion Brun-Baud. Published with permission. Figure is available in color online only.

rupted. 4) The deep portion of the IFOF, with its vertical temporofrontal course, is also interrupted before its termination in the dorsolateral prefrontal cortex, the middle portion of the MFG, the orbitofrontal cortex, and the basal frontal cortex. 5) The uncinate fasciculus, which extends from the medial portion of the temporal lobe to the basal surface of the frontal lobe, is interrupted before its arrival in the orbitofrontal cortex.

\section{Anterior Callosotomy}

The commissural fibers connecting the 2 frontal lobes and forming the forceps minor are disconnected. Posteriorly, in the body of the callosum, the fibers connecting the precentral gyrus are spared.

The anterior commissure is not sectioned because of potential damage to the contralateral hemisphere, either directly or injury to the perforating vessels. Instead, the afferent fibers within this structure are sectioned during the frontobasal disconnection (see below).

\section{Intrafrontal Disconnection}

The superior longitudinal fasciculus and the superior frontooccipital fasciculus, with their horizontal parietofrontal courses, are sectioned, thus interrupting the cortico-cortical afferents from/to the temporal, parietal, and occipital lobes. In particular, both components of the superior longitudinal fasciculus (the indirect and the direct [also called arcuate fasciculus]) are disconnected, thus disconnecting the superior temporal gyrus from the dorsal prefrontal cortex.

Cortico-cortical reciprocal connections to the precentral gyrus from the supplementary motor and premotor areas and prefrontal cortex are also interrupted.

\section{Frontobasal Disconnection}

The dorsal frontal lobe is disconnected from the cingulate gyrus and the paraterminal and paraolfactory areas, thus limiting the afferents from the limbic system, principally the amygdala. This step also obviates the need for sectioning the anterior commissure, which is one of the pathways that connects the inferomesial temporal lobe (amygdaloid nuclei) with its homologous areas in the contralateral hemisphere, crossing the midline just ventral to the supraoptic recess of the third ventricle.

\section{General Considerations}

The aim of the periinsular anterior quadrantotomy is to interrupt the connections of the frontal lobe, while preserving the viability of the parenchyma. The disconnection is performed using bipolar cautery and suction in all stages of the surgery.

During each surgical step, an attempt is made to preserve the large arteries and veins of the cortex to avoid postoperative brain swelling. Careful hemostasis should be performed during the entire procedure, primarily with cottonoids and saline solution, and a drain is left in place in the opened lateral ventricle. This drain can remain for 4-5 days until the CSF is cleared of blood and blood products.

The dura is tightly closed, and the bone flap is repositioned at the end of the procedure with a subgaleal drain.

\section{Illustrative Case}

This male patient was first seen at the age of 44 months for nocturnal left hemiclonic seizures. He had an unremarkable medical and family history. Clinical examination revealed mild hemiparesis associated with brisk deep tendon reflexes on the left side. The patient had mild global developmental delay with language impairment.

Awake and sleep electroencephalography (EEG) showed right frontal spikes and waves and bursts of highamplitude polyspikes $(12 \mathrm{~Hz})$ activated by sleep. Brain MRI showed extensive cortico-subcortical hypertrophic dysplasia involving the right anterior cerebral quadrant, with moderate enlargement of the frontal lobe, a dysmorphic right ventricle, and white matter hyperintensities on 

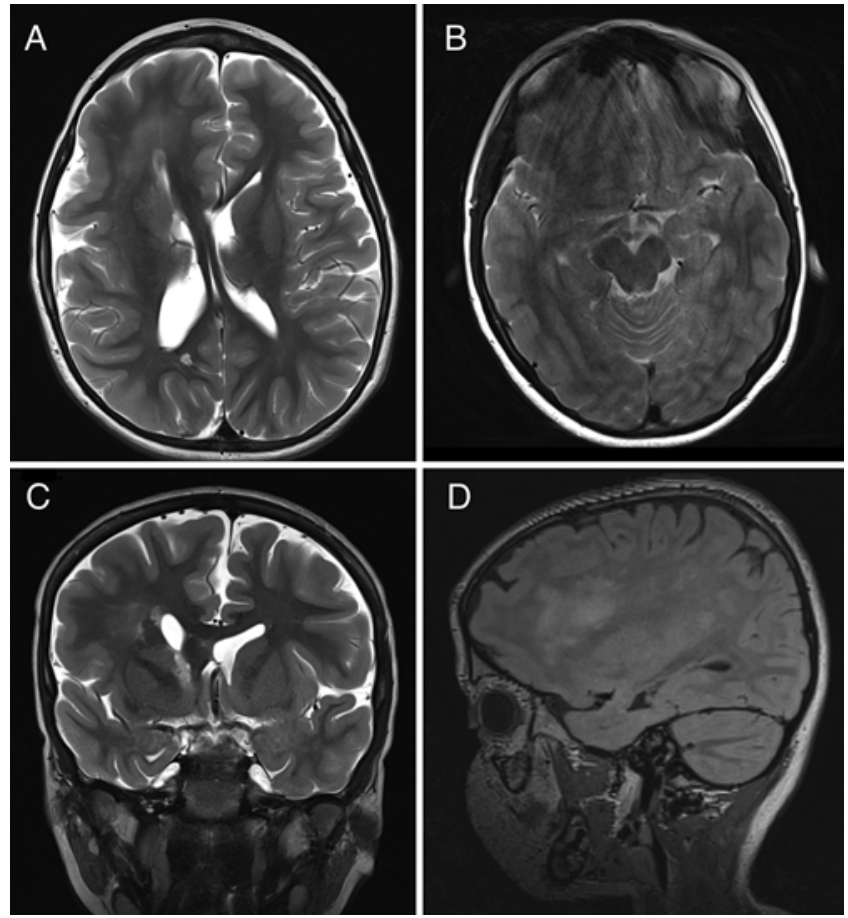

FIG. 5. Axial (A and B), coronal (C), and sagittal (D) MR images of the brain, showing hypertrophic cortical dysplasia (hemimegalencephaly type) in the right anterior quadrant. T2-weighted (A-C) and FLAIR (D) $\mathrm{MRI}$ sequences reveal extensive pachygyria polymicrogyria with thickening of the cortical ribbon with enlargement of the frontal lobe, dysmorphic lateral ventricle, and white matter hyperintensities. The primary motor area was found to be spared.

T2-weighted images. The frontal gyri appeared abnormal, with some portions being simplified and thickened and others with polymicrogyria (Fig. 5). The diagnosis of an anterior hypertrophic cortical dysplasia (hemimegalencephaly type) was made. The primary motor cortex was spared.

The FDG-PET study revealed a more extensive hypometabolism of the right hemisphere, maximal in the right frontal cortex, insula, and basal ganglia (Fig. 6). Highdensity EEG with 256 electrodes suggested 2 right frontal foci: right frontopolar and right frontolateral (Fig. 7). The patient was able to cooperate for the functional MRI study, which revealed that the hand and foot motor cortices were localized posterior to the dysplastic lesion (Fig. 8).

At follow-up, the patient displayed monthly sensorimotor seizures involving the left upper and lower limbs, despite the fact that he was receiving multiple antiepileptic drugs (carbamazepine, lamotrigine, clobazam, levetiracetam), and he progressively developed a minimal hemiparesis on the left side. The patient's parents reported increasing learning difficulties and periods of aggressiveness in an introverted child. EEG findings were very active with continuous spike and waves over the right anterior area. Serial neuropsychological assessments showed low IQ scores (at 7 years: nonverbal IQ 62, verbal IQ 46), with cognitive stagnation and specific language impairment.

When the patient was 9 years old, an anterior periinsu-
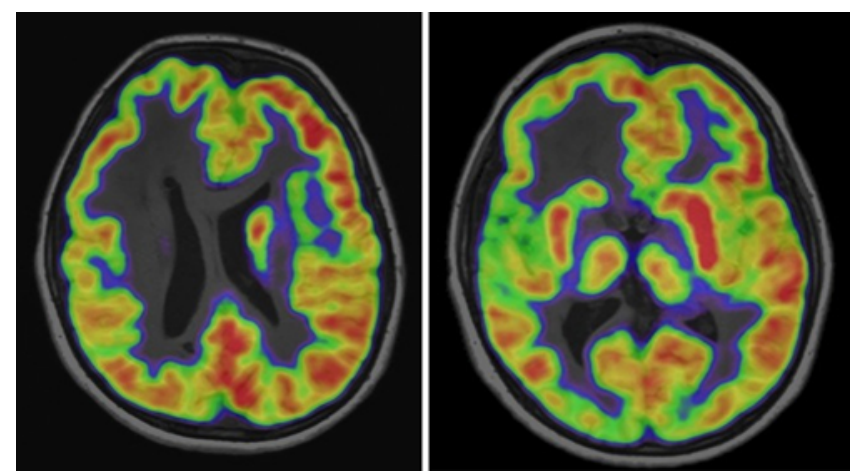

FIG. 6. FDG-PET studies obtained during the interictal phase. Left: Hypometabolism of the right hemisphere, predominantly the frontal lobe, which was more evident at the level of the subcortical structures. Right: Hypometabolism in the right insula. Asymmetry was also observed at the level of the lateral temporal and occipital cortices. Figure is available in color online only.

lar quandrantotomy was performed. Pathological examination revealed focal cortical dysplasia with a complete disorganization of the cortical architecture and no clear border between gray and white matter. The postoperative course was uneventful, and the patient was discharged 5 days after surgery. Brain MRI confirmed a complete frontal lobe disconnection (Fig. 9). Neuropsychological assessment 1 year after surgery revealed significant improvement in language, communication skills, and learning abilities but persistent low IQ scores (nonverbal IQ 52, verbal IQ 53) and weak executive functioning. Three years after surgery, the child is seizure free (Engel Class I), with no motor deficits except for a subtle left dysdiadochokinesia. At 12 years, he attends a specialized school, with ongoing progress in cognitive development. His parents report a very collaborative and calm child.

\section{Discussion}

Extratemporal epilepsy is more frequent in infants, ${ }^{26}$ and intractable epilepsy originating from the frontal lobe accounts for about $20 \%$ of surgical cases. The surgical treatment of this condition, when compared with temporal lobe epilepsy, is more challenging and less well defined..$^{14,15}$ A developmental structural anomaly as a cause of medically intractable epilepsy, such as focal cortical dysplasia, is also more frequent in the pediatric population. ${ }^{13}$

\section{Seizure Outcome}

Results for the surgical treatment of focal cortical dysplasia are variable, especially in cases in which dysplasia extends to an entire lobe or more. The extent of resection seems to be well correlated with a good seizure outcome. ${ }^{16,18-20,22}$ For patients with intractable epilepsy due to lesions involving the posterior quadrant (parietal, occipital, and temporal lobes), Daniel et al. described posterior quadrantic epilepsy surgery and its technical variants, with an Engel Class I in 12 of 13 patients in this series.? D'Agostino et al. reported a similar epileptic outcome in their cohort of 14 patients with posterior quadrantic dys- 


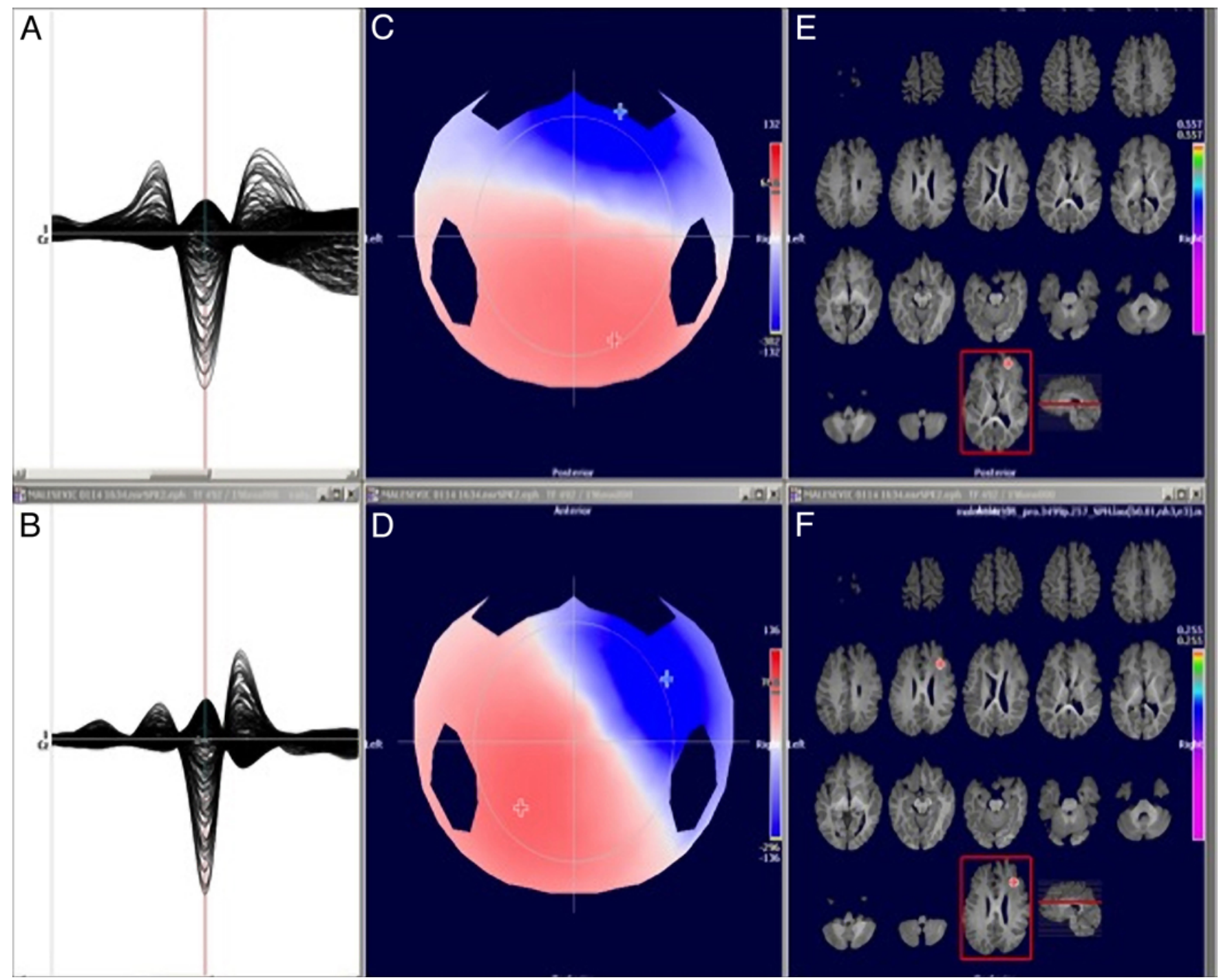

FIG. 7. Electric source imaging of the interictal discharges. Two types of discharges with a slightly different localization were identified. A and B: The plotting of 256 channels superimposed. C and D: Scalp voltage maps. E and F: The calculated intracranial electric peak projected onto the patient's MR image. Maximal interictal activity was observed in the right frontopolar region ( $A, C$, and $\mathrm{E}$ ) and right lateral frontal region (B, D, and F). Figure is available in color online only.

plasia. ${ }^{5}$ Reports in literature dealing specifically with postsurgical seizure outcomes for focal cortical dysplasia of the frontal lobe are scarce.

A meta-analysis performed by Englot et al. reported an overall rate of postoperative favorable seizure outcome (Engel Class I) in $45.1 \%$ of patients with frontal lobe epilepsy of various etiologies and in 54\% of cases of cortical dysplasia. ${ }^{12}$ The predictors of long-term seizure freedom were the presence of abnormal findings on MRI, lesional epilepsy origin, and a localized frontal resection versus a more extended resection. A total resection of the lesion was also associated with an improved outcome compared with subtotal lesionectomy. ${ }^{12}$

Jeha et al. ${ }^{17}$ estimated that with frontal lobectomy the probability of complete seizure freedom was $56 \%$ at 1 year postoperatively, $45 \%$ at 3 years, and $30 \%$ at 5 years. The following factors were identified as independent predictors of seizure recurrence: MRI-negative malformation of cortical development as disease etiology, extrafrontal MRI abnormality, generalized ictal EEG patterns, acute postoperative seizures, and incomplete surgical resection. At the 5-year follow-up, patients were seizure free in $40 \%$ of cases if they had no risk factors, and in $15 \%$ of cases if they did have unfavorable outcome predictors.

\section{Neurological and Functional Outcome}

In the event of a good concordance between the clinical, electrophysiological, and radiological data that delineate an epileptic zone involving the frontal lobe anterior to the motor cortex, a well-performed disconnective surgery (guided by electrophysiology) should not incur any new neurological deficits. In fact, our patient, who had mild hemiparesis prior to surgery, improved after the cessation of seizures. We have to presume that the preoperative mild motor deficit was possibly due to the frequent epileptic at- 

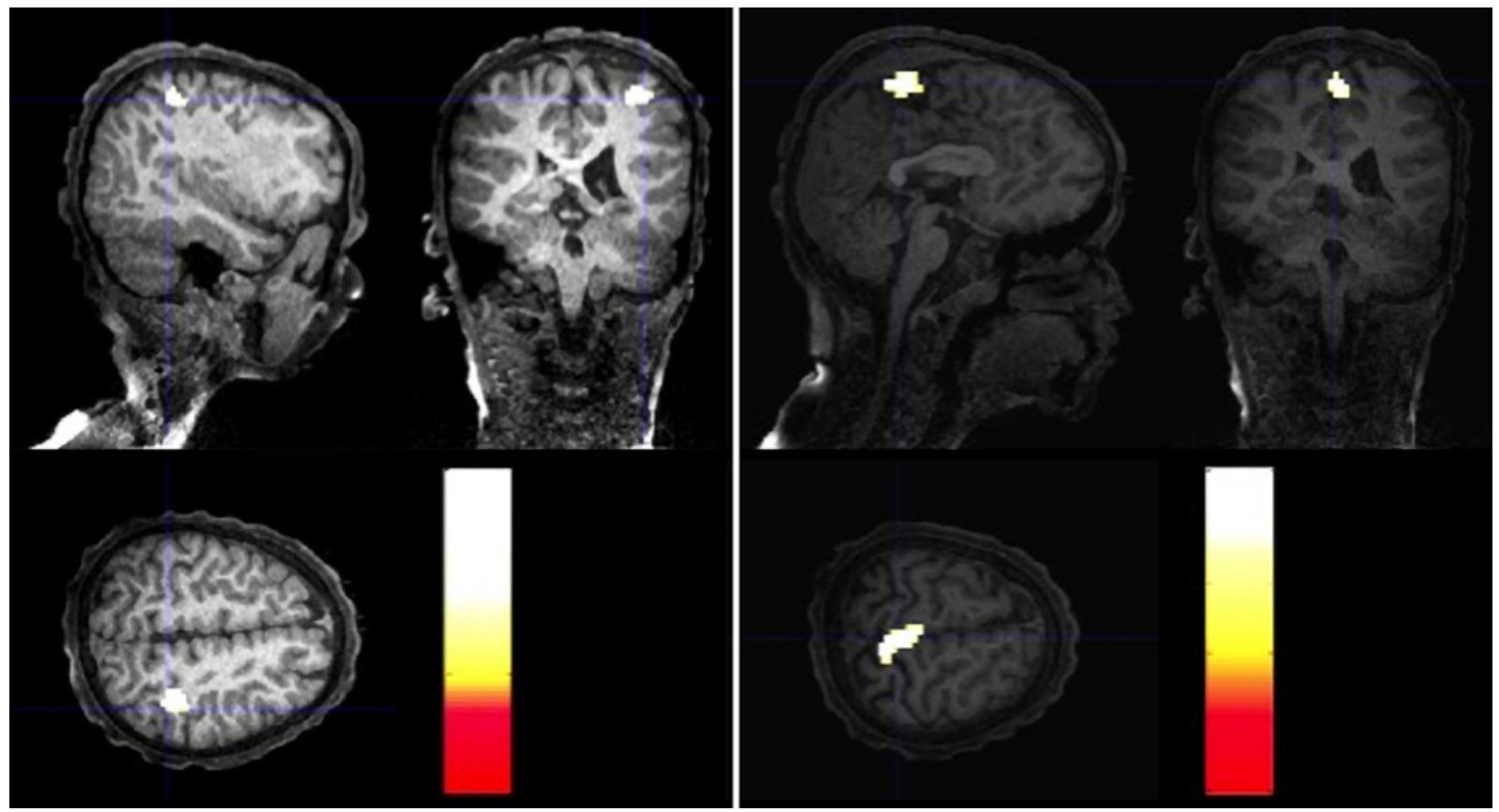

FIG. 8. Functional MR images obtained using a block design of movements versus at rest. Hand (left) and foot (right) motor cortices were localized posterior to the dysplastic cortex in the right frontal lobe. Figure is available in color online only.

tacks and postictal periods. Intellectual deterioration and loss of language properties are rare events after surgery, even after left hemispheric disconnection. ${ }^{11,24}$ Despite poorer global cognitive outcome in children with large dysplasia, possibly due to contralateral or diffuse dysplas- tic changes, an improvement (in gross and fine motor functions, adaptive and personal skills) may be observed in the immediate and long-term postoperative follow-up, ${ }^{11,24}$ as in our case. The duration of epilepsy seems to have an impact on functional outcome, possibly because the seizure activ-
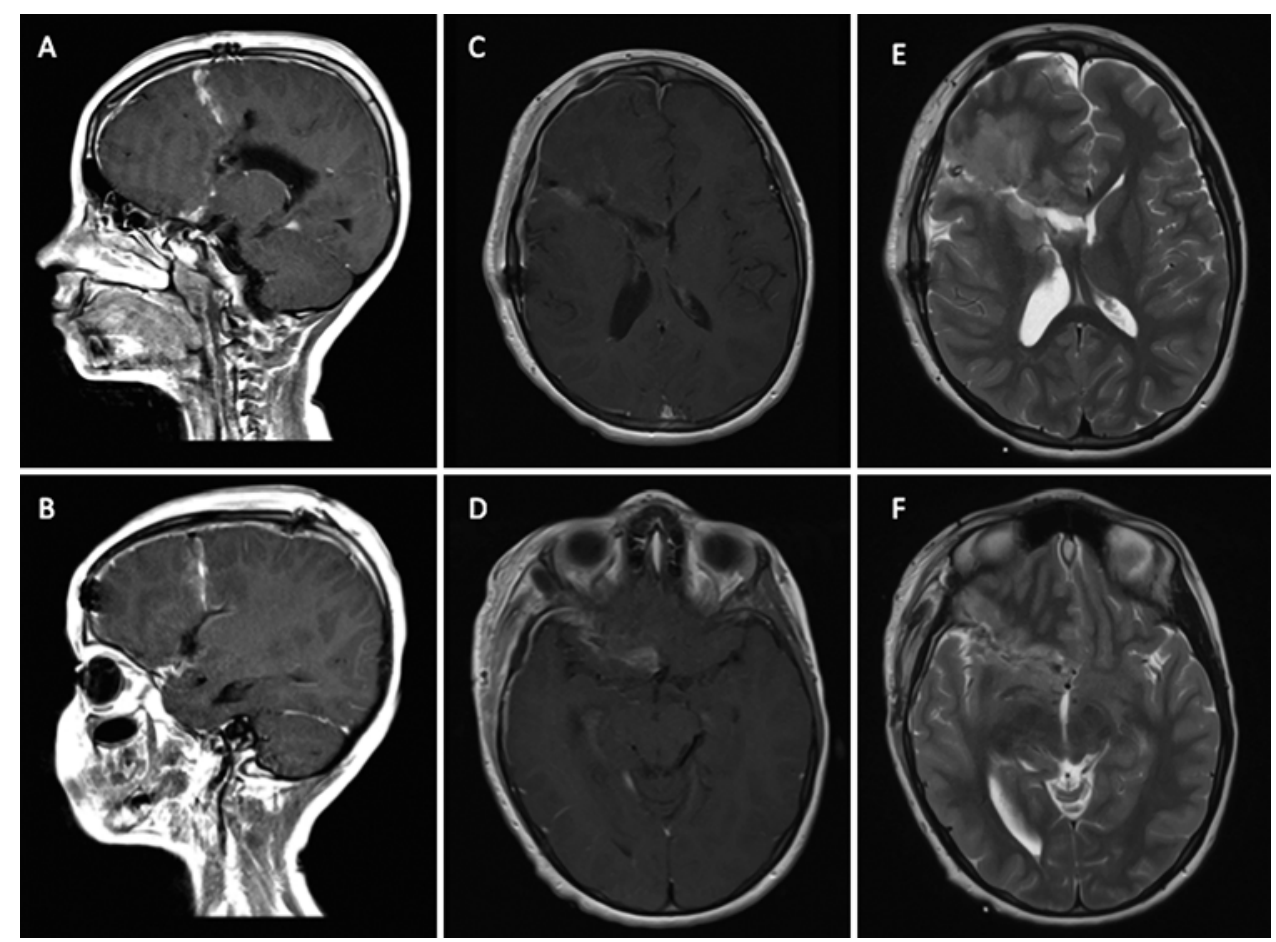

FIG. 9. Postoperative cerebral MR images showing complete disconnection of the right anterior quadrant (frontal lobe). A and B: Sagittal T1-weighted images showing the suprainsular window and intrafrontal disconnection. C-F: Axial T1-weighted (C and D) and T2-weighted ( $E$ and $F$ ) images showing the suprainsular window and intrafrontal disconnection $(\mathrm{C}$ and $\mathrm{E})$ and the frontobasal disconnection ( $\mathrm{D}$ and $\mathrm{F}$ ). 
ity may adversely affect the noninvolved hemisphere. ${ }^{2,11} \mathrm{~A}$ younger age at surgery may be a positive prognostic factor for postoperative adaptive functioning, likely because of the greater plasticity in early life. ${ }^{2}$

\section{Potential Pitfalls and Complication Avoidance}

A complete disconnection of the entire epileptic zone is key to the entire procedure, and attention should be paid to the correct execution of all surgical steps. There are some pitfalls to avoid while performing an anterior quadrantotomy, as detailed below.

During the suprainsular window, the vessels and the insular cortex should be respected as much as possible to limit the risk of hemorrhagic or ischemic complications and of a new postoperative deficit (loss of preexisting motor function).

During the anterior callosotomy, the incision at the junction between the roof of the lateral ventricle and the septum pellucidum should be strictly performed in a parasagittal direction. If the cut is too oblique, there is a risk of entering the contralateral cingulate gyrus and frontal lobe. Care should be taken to perform a complete disconnection of the anterior corpus callosum, including the genu and the rostrum, while sparing the interhemispheric commissural fibers from the motor cortex located posteriorly. The arachnoid of the pericallosal cistern and the pericallosal arteries should be used as protection and guidance for the callosotomy, with care to preserve the integrity of these arteries and their branches.

During the frontobasal disconnection, there is a risk of performing the incision too anteriorly, thus leaving some functional areas posteriorly with a possible spreading of the epileptogenic discharges. ${ }^{21}$ The resection plane should follow the sphenoid ridge and terminate just anterior to the carotid bifurcation.

The presence of distorted anatomy (in cases of hypertrophic dysplasia) may further complicate the intraoperative evaluation of a complete disconnection. This is probably one of the reasons for the worse postsurgical seizure outcome with hemimegalencephaly and its variants when compared with other pathologies in hemispherotomy series. ${ }^{3,4,11,18,23}$

\section{Advantages of Disconnective Surgery}

The standard approach for large frontal lobe epileptic lesions is resective surgery. However, this procedure consists of removing a large amount of tissue, with all the possible long-term complications linked to the cavity effect. These are well-described complications seen after resective surgeries for hemispheric epilepsy. ${ }^{6,79,10}$ Similar to the evolution of disconnective procedures for hemispheric and posterior quadrantic surgeries, this procedure of an anterior quadrantotomy is a logical evolution along the same lines. ${ }^{7,8,25}$ The introduction of disconnective techniques in epilepsy surgery has also allowed the reduction of craniotomy size, duration of surgery, and hospital stay (related to an easier early postoperative period). Anterior quadrantotomy should thereby eliminate the complications associated with a large brain resection similar to that seen in hemispherectomies and large lobar resections.

\section{Conclusions}

The periinsular anterior quadrantotomy represents an excellent option for the treatment of patients with refractory epilepsy restricted to one frontal lobe and associated with normal or near-normal motor function.

The preservation of motor areas is one of the key steps of this procedure, and intraoperative functional mapping is necessary to perform and guide the disconnection. In the presence of a concordance of data from clinical, radiological, and EEG evaluations, the seizure outcome of this procedure is expected to be similar to other forms of disconnective surgery for subhemispheric and hemispheric epilepsy syndromes. Further studies in larger patient cohorts will be necessary to confirm these preliminary findings.

\section{Acknowledgments}

We thank Prof. Jean-Guy Villemure, former head of our department and pioneer of disconnective pediatric epilepsy surgery, for mentoring and guiding our epilepsy surgery program. We would also like to express our gratitude to Mrs. Marion Brun, of the Department of Otorhinolaryngology at the University Hospital of Lausanne, for the creation of the diagrammatical images of the procedure.

\section{References}

1. Babu KS, Chandy MJ: Reliability of somatosensory evoked potentials in intraoperative localization of the central sulcus in patients with perirolandic mass lesions. Br J Neurosurg 11:411-417, 1997

2. Basheer SN, Connolly MB, Lautzenhiser A, Sherman EM, Hendson G, Steinbok P: Hemispheric surgery in children with refractory epilepsy: seizure outcome, complications, and adaptive function. Epilepsia 48:133-140, 2007

3. Bulteau C, Dorfmüller G, Fohlen M, Jalin C, Oliver MV, Delalande O: [Long-term outcome after hemispheric disconnection.] Neurochirurgie 54:358-361, 2008 (Fr)

4. Carreño M, Wyllie E, Bingaman W, Kotagal P, Comair Y, Ruggieri P: Seizure outcome after functional hemispherectomy for malformations of cortical development. Neurology 57:331-333, 2001

5. D'Agostino MD, Bastos A, Piras C, Bernasconi A, Grisar T, Tsur VG, et al: Posterior quadrantic dysplasia or hemihemimegalencephaly: a characteristic brain malformation. Neurology 62:2214-2220, 2004

6. Daniel RT, Joseph TP, Gnanamuthu C, Chandy MJ: Hemispherotomy for paediatric hemispheric epilepsy. Stereotact Funct Neurosurg 77:219-222, 2001

7. Daniel RT, Meagher-Villemure K, Farmer JP, Andermann F, Villemure JG: Posterior quadrantic epilepsy surgery: technical variants, surgical anatomy, and case series. Epilepsia 48:1429-1437, 2007

8. Daniel RT, Meagher-Villemure K, Roulet E, Villemure JG: Surgical treatment of temporoparietooccipital cortical dysplasia in infants: report of two cases. Epilepsia 45:872-876, 2004

9. Daniel RT, Thomas SG, Thomas M: Role of surgery in pediatric epilepsy. Indian Pediatr 44:263-273, 2007

10. Daniel RT, Villemure JG: Peri-insular hemispherotomy: potential pitfalls and avoidance of complications. Stereotact Funct Neurosurg 80:22-27, 2003

11. Devlin AM, Cross JH, Harkness W, Chong WK, Harding B, Vargha-Khadem F, et al: Clinical outcomes of hemispherectomy for epilepsy in childhood and adolescence. Brain 126:556-566, 2003 
12. Englot DJ, Wang DD, Rolston JD, Shih TT, Chang EF: Rates and predictors of long-term seizure freedom after frontal lobe epilepsy surgery: a systematic review and meta-analysis. J Neurosurg 116:1042-1048, 2012

13. Farrell MA, DeRosa MJ, Curran JG, Secor DL, Cornford ME, Comair YG, et al: Neuropathologic findings in cortical resections (including hemispherectomies) performed for the treatment of intractable childhood epilepsy. Acta Neuropathol 83:246-259, 1992

14. Fuentes A, Smith ML: Patterns of verbal learning and memory in children with intractable temporal lobe or frontal lobe epilepsy. Epilepsy Behav 53:58-65, 2015

15. Garcia PA, Laxer KD: Lateral frontal lobe epilepsies in Lüders HO, Comair YG (eds): Epilepsy Surgery, ed 2. Philadelphia: Lippincott Williams and Wilkins, 2001, pp 111-134

16. Hirabayashi S, Binnie CD, Janota I, Polkey CE: Surgical treatment of epilepsy due to cortical dysplasia: clinical and EEG findings. J Neurol Neurosurg Psychiatry 56:765-770, 1993

17. Jeha LE, Najm I, Bingaman W, Dinner D, Widdess-Walsh P, Lüders H: Surgical outcome and prognostic factors of frontal lobe epilepsy surgery. Brain 130:574-584, 2007

18. Kloss S, Pieper T, Pannek H, Holthausen H, Tuxhorn I: Epilepsy surgery in children with focal cortical dysplasia (FCD): results of long-term seizure outcome. Neuropediatrics 33:21-26, 2002

19. Krsek P, Maton B, Jayakar P, Dean P, Korman B, Rey G, et al: Incomplete resection of focal cortical dysplasia is the main predictor of poor postsurgical outcome. Neurology 72:217-223, 2009

20. Leiphart JW, Peacock WJ, Mathern GW: Lobar and multilobar resections for medically intractable pediatric epilepsy. Pediatr Neurosurg 34:311-318, 2001

21. Mittal S, Farmer JP, Rosenblatt B, Andermann F, Montes JL, Villemure JG: Intractable epilepsy after a functional hemispherectomy: important lessons from an unusual case. Case report. J Neurosurg 94:510-514, 2001
22. Palmini A, Gambardella A, Andermann F, Dubeau F, da Costa JC, Olivier A, et al: Operative strategies for patients with cortical dysplastic lesions and intractable epilepsy. Epilepsia 35 (Suppl 6):S57-S71, 1994

23. Thomas SG, Chacko AG, Thomas MM, Babu KS, Russell PS, Daniel RT: Outcomes of disconnective surgery in intractable pediatric hemispheric and subhemispheric epilepsy. Int J Pediatr 2012:527891, 2012

24. Thomas SG, Daniel RT, Chacko AG, Thomas M, Russell PS: Cognitive changes following surgery in intractable hemispheric and sub-hemispheric pediatric epilepsy. Childs Nerv Syst 26:1067-1073, 2010

25. Villemure JG, Daniel RT: Peri-insular hemispherotomy in paediatric epilepsy. Childs Nerv Syst 22:967-981, 2006

26. Wyllie E, Comair YG, Kotagal P, Raja S, Ruggieri P: Epilepsy surgery in infants. Epilepsia 37:625-637, 1996

\section{Disclosures}

The authors report no conflict of interest concerning the materials or methods used in this study or the findings specified in this paper.

\section{Author Contributions}

Conception and design: Daniel. Acquisition of data: all authors. Analysis and interpretation of data: Daniel, Cossu, Seeck, Pralong. Drafting the article: Daniel, Cossu, Lebon. Critically revising the article: Daniel, Cossu, Lebon, Seeck, Messerer, Roulet-Perez. Approved the final version of the manuscript on behalf of all authors: Daniel. Study supervision: Daniel.

\section{Correspondence}

Roy Thomas Daniel, Department of Neurosurgery, University Hospital of Lausanne, Rue du Bugnon 46, Lausanne 1011, Switzerland. email: roy.daniel@chuv.ch. 